\title{
Limit Cycles of a Class of Perturbed Differential Systems via the First-Order Averaging Method
}

\author{
Amor Menaceur, ${ }^{1}$ Salah Mahmoud Boulaaras ${ }^{(D,}{ }^{2,3}$ Amar Makhlouf, ${ }^{4}$ \\ Karthikeyan Rajagobal $\mathbb{D}^{5},{ }^{5}$ and Mohamed Abdalla $\mathbb{D}^{6,7}$ \\ ${ }^{1}$ Laboratory of Analysis and Control of Differential Equations "ACED", Departement of Mathematics, University of Guelma, \\ P.O. Box 401, Guelma 24000, Algeria \\ ${ }^{2}$ Department of Mathematics, College of Sciences and Arts ArRass, Qassim University, AI-Qassim, Saudi Arabia \\ ${ }^{3}$ Laboratory of Fundamental and Applied Mathematics of Oran (LMFAO), University of Oran 1, Ahmed Benbella, Algeria \\ ${ }^{4}$ Departement of Mathematics, Annaba University, Box. 12 El Hadjar, Annaba, Algeria \\ ${ }^{5}$ Center for Nonlinear Systems Chennai Institute of Technology, Chennai, India \\ ${ }^{6}$ Mathematics Department, College of Science, King Khalid University, Abha 61413, Saudi Arabia \\ ${ }^{7}$ Mathematics Department, Faculty of Science, South Valley University, Qena 83523, Egypt
}

Correspondence should be addressed to Karthikeyan Rajagobal; rkarthiekeyan@gmail.com

Received 7 February 2021; Revised 25 February 2021; Accepted 4 March 2021; Published 19 March 2021

Academic Editor: Heng Liu

Copyright (c) 2021 Amor Menaceur et al. This is an open access article distributed under the Creative Commons Attribution License, which permits unrestricted use, distribution, and reproduction in any medium, provided the original work is properly cited.

By means of the averaging method of the first order, we introduce the maximum number of limit cycles which can be bifurcated from the periodic orbits of a Hamiltonian system. Besides, the perturbation has been used for a particular class of the polynomial differential systems.

\section{Introduction}

As we know that the second part of the 16 Hilbert problem $([1,2])$ wants to find a uniform upper bound for the number of limit cycles of all polynomial differential systems of a given degree, we refer the readers to see $[3,4]$. The limit cycles problem and the center problem are fastened on specified classes of systems. For instance, we refer to Kukles systems (see, for example, [5-9]) and Liénard systems given by

$$
\left\{\begin{array}{l}
\dot{u}=v, \\
\dot{v}=-u-f(u),
\end{array}\right.
$$

where $f(u)$ is a polynomial in the variable $u$ of degree $m$. For these systems, in 1977, Lins et al. [10] presented the conjecture that if $f(u)$ has degree $m \geq 1$, then system (1) has at most $[m / 2]$ limit cycles where [.] denotes the integer part function. They prove this conjecture for $m=1,2$. The conjecture for $m=3$ has been proved recently by Chengzi and Llibre in [11].

Suppose that polynomials $f(u)$ and $g(u)$ are in the variable $u$ of degrees $n$ and $m$, respectively; then, Llibre et al. [12] established the following generalized Liénard polynomial differential system:

$$
\left\{\begin{array}{l}
\dot{u}=v, \\
\dot{v}=-g(u)-f(u) v,
\end{array}\right.
$$

where $[n+m-1 / 2]$ is limit cycles.

Llibre and Makhlouf [13] studied the number of limit cycles of the following generalized Liénard polynomial differential system:

$$
\left\{\begin{array}{l}
\dot{u}=-v^{2 p-1}, \\
\dot{v}=u^{2 q-1}-\epsilon f(u) v^{2 n-1},
\end{array}\right.
$$


where $f(u)$ is a polynomial of degree $m, p, q$, and $n$ are positive integers, and $\epsilon$ is a small parameter.

They introduced the following theorem.

Theorem 1 (see [13]). Let $m$ be the degree of the polynomial $f(u)$, and $\epsilon \neq 0$; then, the polynomial differential system (3) can have at least $[\mathrm{m} / 2]$ limit cycles.

Also, Jianyuan and Shuliang [14] investigated the maximum number of limit cycles of the following polynomial differential system:

$$
\left\{\begin{array}{l}
\dot{u}=-v^{2 p-1}, \\
\dot{v}=u^{2 m p-1}+\epsilon\left(p u^{2 m p}+m p v^{2 p}\right)(g(u, v)-A),
\end{array}\right.
$$

where $\epsilon$ is a small parameter, $A>0, p, m \in \mathbb{N}$, and $g(u, v)$ is a polynomial of degree $n$ with $g(0,0)=0$.

In this manuscript, we discuss the maximum number of limit cycles of the following polynomial differential system:

$$
\left\{\begin{array}{l}
\dot{u}=-v^{2 p-1}-\epsilon p u f(u, v), \\
\dot{v}=u^{2 q-1}-\epsilon q v f(u, v),
\end{array}\right.
$$

where $f(u, v)$ is a polynomial of degree $m, \epsilon$ is a small parameter, and $p, q \in \mathbb{N}$. Clearly, system (5) with $\epsilon=0$ is a Hamiltonian system with Hamiltonian

$$
H(u, v)=\frac{1}{2 q} u^{2 q}+\frac{1}{2 p} v^{2 p} .
$$

More precisely, our main results are the following.

Theorem 2. For the sufficiently small $|\varepsilon|$, system (5) has at most

$$
\lambda=\left[\frac{m}{2}\right] \max \{p, q\}
$$

limit cycles bifurcating from the periodic orbits of the center $\dot{u}=-v^{2 p-1}, \dot{v}=u^{2 q-1}$, by using the averaging theory of first order.

The proof of Theorem 2 is given in Section 3.

Theorem 3. Consider system (5) with $q=n p$, where $n$ is a positive integer; then, for $|\varepsilon|$ sufficiently small, the maximum number of limit cycles of the polynomial differential system (5) bifurcating from the periodic orbits of the center $\dot{u}=-v^{2 p-1}, \dot{v}=$ $u^{2 n p-1}$ using the averaging theory of first order is

$$
\begin{array}{ll}
\text { (a). } \mu_{1}=\frac{1}{2}\left(\left[\frac{m}{2}\right]\left(\left[\frac{m}{2}\right]+3\right)\right), & \text { if }\left[\frac{m}{2}\right] \leq n-1, \\
\text { (b). } \mu_{2}=n\left[\frac{m}{2}\right]-\frac{n(n-3)+2}{2}, & \text { if }\left[\frac{m}{2}\right] \geq n .
\end{array}
$$

The proof of Theorem 3 is given in Section 4. Also, an example is given with its limit cycles (see Figure 1).

\section{First-Order Averaging Method}

Here, we state the basic outcomes from the averaging theory of first order, which will be used to prove the main outcomes.

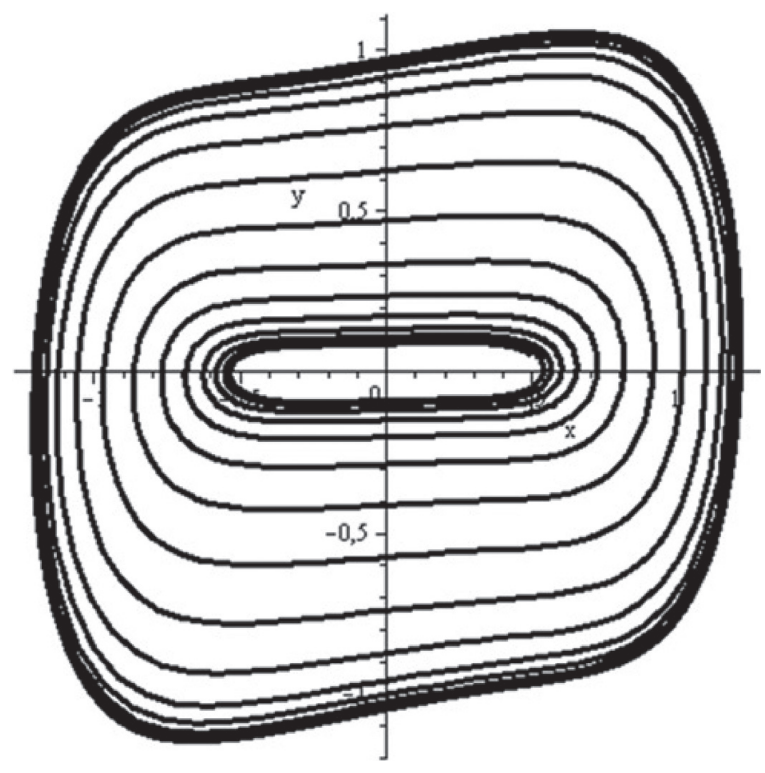

FIGURE 1: Limit cycles for the system in Example 1 with $\epsilon=0.01$.

Theorem 4. Consider the following two initial-value problems:

$$
\begin{aligned}
\dot{u} & =\epsilon R(t, u)+\epsilon^{2} G(t, u, \epsilon), \\
u(0) & =u_{0}, \\
\dot{v} & =\epsilon f^{0}(v), \\
v(0) & =u_{0},
\end{aligned}
$$

where $u, v$ and $u_{0} \in D$ which is an open domain of $\mathbb{R}$, $t \in[0, \infty), \epsilon \in\left(0, \epsilon_{0}\right], R$ and $G$ are periodic functions with their period $T$ with its variable $t$, and $f^{0}(v)$ is the average function of $R(t, v)$ with respect to $t$, that is,

$$
f^{0}(v)=\frac{1}{T} \int_{0}^{T} R(t, v) \mathrm{d} t .
$$

Assume that

(i) $R, \partial R / \partial u, \partial^{2} R / \partial u^{2}, G$, and $\partial G / \partial u$ are well defined, continuous, and bounded by a constant independent by $\epsilon \in\left(0, \epsilon_{0}\right]$ in $[0, \infty) \times D$.

(ii) $T$ is a constant independent of $\epsilon$.

(iii) $v(t)$ belongs to $D$ on the time scale $1 / \epsilon$. Then, the following statements hold:

(a) On the time scale $1 / \epsilon$, we have

$$
u(t)-v(t)=O(\epsilon), \text { as } \epsilon \longrightarrow 0
$$

(b) If $p$ is an equilibrium point of the averaged system (10) such that

$$
\left.\frac{\partial f^{0}}{\partial v}\right|_{v=p} \neq 0,
$$

then system (9) has a $T$-periodic solution $\phi(t, \epsilon) \longrightarrow p$ as $\epsilon \longrightarrow 0$ 
(c) If (13) is negative, the corresponding periodic solution $\phi(t, \epsilon)$ of equation (9) according to $(t, u)$ is asymptotically stable for all $\epsilon$ sufficiently small; if (13) is positive, then it is unstable

For more information about the averaging theory, see, e. g., $[15-17]$.

\section{Proof of Theorem 2}

The (p.q)-trigonometrical functions were defined by Liapunov [18]. Let $u(\theta)=\operatorname{Cs} \theta$ and $v(\theta)=\operatorname{Sn} \theta$ be the solution of the following initial value problem:

$$
\begin{aligned}
\dot{u} & =-v^{2 p-1}, \\
\dot{v} & =u^{2 q-1}, \\
u(0) & =\sqrt[2]{q} \frac{1}{p} \text { and } v(0)=0 .
\end{aligned}
$$

Furthermore, the following properties are satisfied:

(a) The functions $\operatorname{Cs} \theta$ and $\operatorname{Sn} \theta$ are $T$-periodic with

$$
T=2 p^{(-1 / 2 q)} q^{(-1 / 2 p)} \frac{\Gamma(1 / 2 p) \Gamma(1 / 2 q)}{\Gamma((1 / 2 p)+(1 / 2 q))},
$$

where $\Gamma$ is the gamma function.

(b) For $p=q=1$, we have $\operatorname{Cs} \theta=\cos \theta$ and $\operatorname{Sn} \theta=\sin \theta$

(c) $p \operatorname{Cs}^{2 p} \theta+q \operatorname{Sn}^{2 q} \theta=1$

(d) Let $\operatorname{Cs} \theta$ and $\sin \theta$ be the (1.q)-trigonometrical functions, when $i$ and $j$ are both even (see [19])

$$
\int_{0}^{T} C s^{i} \theta S n^{j} \theta \mathrm{d} \theta=2 q^{-(j+1 / 2)} \frac{\Gamma(i+1 / 2 q) \Gamma(j+1 / 2)}{\Gamma((i+1 / 2 q)+(j+1 / 2))} .
$$

We shall need the first-order averaging theory to prove Theorem 2. We write system (5) in $(p, q)$-polar coordinates $(r, \theta)$, where $u=r^{p} \operatorname{Cs} \theta$ and $v=r^{q} \operatorname{Sn} \theta$. In this way, system (5) will become written in the standard form for applying the averaging theory. If we write $f(u, v)=\sum_{i+j=0}^{m} a_{i, j} u^{i} v^{j}$, then system (5) becomes

$$
\left\{\dot{r}=-\epsilon r \sum_{i+j=0}^{m}\left(a_{i, j} r^{p i+q j} C s^{i} \theta S n^{j} \theta\right)\right), \dot{\theta}=r^{2 p q-p-q} .
$$

Treating $\theta$ as the independent variable, we get from system (17) the following:

$$
\frac{\mathrm{d} r}{\mathrm{~d} \theta}=\epsilon R(r, \theta)
$$

where

$$
R(r, \theta)=-r^{-2 p q+p+q+1} \sum_{i+j=0}^{m}\left(a_{i, j} r^{p i+q j} C s^{i} \theta S n^{j} \theta\right) .
$$

By using the notation which is introduced in Section 2, we get

$$
f^{0}(r)=-\frac{r^{-2 p q+p+q+1}}{T} \sum_{i+j=0}^{m}\left(a_{i, j} p^{p i+q j} \int_{0}^{T} C s^{i} \theta S n^{j} \theta \mathrm{d} \theta\right),
$$

and we write

$$
f^{0}(r)=-\frac{r^{-2 p q+p+q+1}}{T} \sum_{i+j=0}^{m} a_{i, j} \alpha_{i, j} r^{p i+q j},
$$

where

$$
\alpha_{i, j}=\int_{0}^{T} C s^{i} \theta S n^{j} \theta \mathrm{d} \theta .
$$

It is known that

$$
\begin{aligned}
& \alpha_{i, j}=0, \text { if } i \text { or } j \text { is odd, } \\
& \alpha_{i, j}>0, \text { if } i \text { and } j \text { are even. }
\end{aligned}
$$

Hence,

$$
f^{0}(r)=-\frac{r^{-2 p q+p+q+1}}{T} \sum_{s+k=0}^{[m / 2]} a_{2 s, 2 k} \alpha_{2 s, 2 k} r^{2(p s+q k)} .
$$

For the simplicity of calculation, let $A_{s, k}=a_{2 s, 2 k} \alpha_{2 s, 2 k}$; therefore, (24) can be reduced to

$$
f^{0}(r)=-\frac{r^{-2 p q+p+q+1}}{T} \sum_{s+k=0}^{[m / 2]} A_{s, k} r^{2(p s+q k)} .
$$

The positive zeros number of $f^{0}(r)$, as we know, is equal to the following:

$$
K(r)=\sum_{s+k=0}^{[m / 2]} A_{s, k} r^{2(p s+q k)},
$$

and then, to find the real positive roots of $K(r)$, we must find the zeros of a polynomial in the variable $t=r^{2}$ :

$$
H(t)=\sum_{s+k=0}^{[m / 2]} A_{s, k} t^{p s+q k} .
$$

Now, we stretch the polynomial (27) as follows:

$$
\begin{aligned}
H(t)= & A_{0,0}+A_{1,0} t^{p}+A_{0,1} t^{q}+A_{2,0} t^{2 p}+A_{1,1} t^{p+q}+A_{0,2} t^{2 q}+\cdots+A_{l, 0} t^{l p}+A_{l-1,1} t^{(l-1) p+q}+A_{l-2,2} t^{(l-2) p+2 q} \\
& +\cdots+A_{2, l-2} t^{2 p+(l-2) q}+A_{1, l-1} t^{p+(l-1) q}+A_{0, l} t^{q l}+\cdots+A_{[m / 2], 0} t^{[m / 2] p}+A_{[m / 2]-1,1} t^{([m / 2]-1) p+q}+A_{[m / 2]-2,2} t^{([m / 2]-2) p+2 q} \\
& +\cdots+A_{2,[m / 2]-2} t^{2 p+([m / 2]-2) q}+A_{1,[m / 2]-1} t^{t^{+([m / 2]-1) q}}+A_{0,[m / 2]} t^{[m / 2] q} .
\end{aligned}
$$


So, the degree of $H(t)$ is bounded by $\lambda=[m / 2] \max \{p, q\}$, and we conclude that $f^{0}(r)$ has at most $\lambda$ positive root $r$. Hence, Theorem 2 is proved.

\section{Proof of Theorem 3}

Consider the polynomial differential system (5) with $q=\mathrm{np}$. From equation (25), we obtain

$$
f^{0}(r)=-\frac{r^{n p(-2 p+1)+p+1}}{T} \sum_{s+k=0}^{[m / 2]} A_{s, k} r^{2 p(s+n k)} .
$$

The zeros positive number of $f^{0}(r)$ is equal to the following:

$$
S(r)=\sum_{s+k=0}^{[m / 2]} A_{s, k} r^{2 p(s+n k)}
$$

We write (30) as follows:

$$
\begin{aligned}
& S(r)=A_{0,0}+\left(A_{1,0} r^{2 p}+A_{0,1} r^{2 p n}\right)+\left(A_{2,0} r^{4 p}+A_{1,1} r^{(n+1) 2 p}+A_{0,2} r^{4 n p}\right)+\left(A_{3,0} r^{6 p}+A_{2,1} r^{(n+2) 2 p}+A_{1,2} r^{(1+2 n) 2 p}+A_{0,3} r^{6 n p}\right) \\
& \cdot\left(\left(A_{4,0} r^{8 p}+A_{3,1} r^{(n+3) 2 p}+A_{2,2} r^{(2+2 n) 2 p}+A_{1,3} r^{(1+3 n) 2 p}+A_{0,4} r^{8 n p}\right)+\cdots\right. \\
& +\left[\begin{array}{c}
A_{[m / 2]-2,0} r^{([m / 2]-2) 2 p}+A_{[m / 2]-3,1} r^{([m / 2]+n-3) 2 p}+A_{[m / 2]-4,2} r^{([m / 2]+2 n-4) 2 p}+\cdots+A_{2,[m / 2]-4} r^{(2+([m / 2]-4) n) 2 p} \\
+A_{1,[m / 2]-3} r^{(1+([m / 2]-3) n) 2 p}+A_{0,([m / 2]-2)} r^{([m / 2]-2) 2 n p}
\end{array}\right] \\
& +\left[\begin{array}{c}
A_{[m / 2]-1,0} r^{([m / 2]-1) 2 p}+A_{[m / 2]-2,1} r^{([m / 2]+n-2) 2 p}+A_{[m / 2]-3,2} r^{([m / 2]+2 n-3) 2 p}+\cdots+A_{2,[m / 2]-3} r^{(2+([m / 2]-3) n) 2 p} \\
+A_{1,[m / 2]-2} r^{(1+([m / 2]-2) n) 2 p}+A_{0,[m / 2]-1} r^{([m / 2]-1) 2 n p}
\end{array}\right] \\
& +\left[\begin{array}{c}
A_{[m / 2], 0} r^{[m / 2] 2 p}+A_{[m / 2]-1,1} r^{([m / 2]+n-1) 2 p}+A_{[m / 2]-2,2} r^{([m / 2]+2 n-2) 2 p}+\cdots+A_{2,[m / 2]-2} r^{(2+([m / 2]-2) n) 2 p} \\
+A_{1,[m / 2]-1} r^{(1+([m / 2]-1) n) 2 p}+A_{0,[m / 2]} r^{[m / 2] 2 n p}
\end{array}\right] .
\end{aligned}
$$

Let us write (31) as

$$
\begin{aligned}
S(r)= & {\left[A_{0,0}+A_{1,0} r^{2 p}+A_{2,0} r^{4 p}+\cdots+A_{[m / 2]-2,0} r^{([m / 2]-2) 2 p}+A_{[m / 2]-1,0} r^{([m / 2]-1) 2 p}+A_{[m / 2], 0} r^{[m / 2] 2 p}\right]+} \\
& \cdot\left[A_{0,1} r^{2 n p}+A_{1,1} r^{(n+1) 2 p}+A_{2,1} r^{(n+2) 2 p}+\cdots+A_{[m / 2]-3,1} r^{(n+[m / 2]-3) 2 p}\right. \\
& \left.+A_{[m / 2]-2,1} r^{(n+[m / 2]-2) 2 p}+A_{[m / 2]-1,1} r^{(n+[m / 2]-1) 2 p}\right] \\
& +\left[A_{0,2} r^{4 n p}+A_{1,2} r^{(2 n+1) 2 p}+A_{2,2} r^{(2 n+2) 2 p}+\cdots+A_{[m / 2]-4,2} r^{(2 n+[m / 2]-4) 2 p}+A_{[m / 2]-3,2} r^{(2 n+[m / 2]-3) 2 p}\right. \\
& \left.+A_{[m / 2]-2,2} r^{(2 n+[m / 2]-2) 2 p}\right] \\
& +\cdots+\left[A_{0,([m / 2]-2)} r^{(([m / 2]-2) n) 2 p}+A_{1,[m / 2]-2} r^{(1+([m / 2]-2) n) 2 p}+A_{2,[m / 2]-2} r^{(2+([m / 2]-2) n) 2 p}\right] \\
& +\left[A_{0,[m / 2]-1} r^{([m / 2]-1) 2 n p}+A_{1,[m / 2]-1} r^{(1+([m / 2]-1) n) 2 p}\right] \\
& +A_{0,[m / 2]} r^{[m / 2] n} .
\end{aligned}
$$


To find the number of positive roots of polynomials $S(r)$, we distinguish two cases.

Case 1. For $[m / 2] \leq n-1$, the number term in polynomial (32) is

$$
\begin{aligned}
\left(\left[\frac{m}{2}\right]+1\right) & +\left[\frac{m}{2}\right]+\left(\left[\frac{m}{2}\right]-1\right) \\
& +\cdots+2+1=\frac{1}{2}\left(\left[\frac{m}{2}\right]+2\right)\left(\left[\frac{m}{2}\right]+1\right) .
\end{aligned}
$$

Now, the Descartes theorem of the Appendix will be applied, and the appropriate coefficients $A_{i, j}$ can be selected for the simple positive zeros number of $S(r)$ as at most

$$
\mu_{1}=\frac{1}{2}\left(\left[\frac{m}{2}\right]+2\right)\left(\left[\frac{m}{2}\right]+1\right)-1=\frac{1}{2}\left(\left[\frac{m}{2}\right]\left(\left[\frac{m}{2}\right]+3\right)\right) .
$$

Hence, (a) of Theorem 3 is proved.

Case 2. For $[m / 2] \geq n$, the number term in polynomial (32) is

$$
\begin{array}{r}
\left(\left[\frac{m}{2}\right]+1\right)+\left[\frac{m}{2}\right]+\left(\left[\frac{m}{2}\right]-1\right)+\cdots+2+1-\left(\left[\frac{m}{2}\right]-n+1\right)-\left(\left[\frac{m}{2}\right]-n\right)-\left(\left[\frac{m}{2}\right]-n-1\right)-\cdots-2-1 \\
=\frac{1}{2}\left[\left(\left[\frac{m}{2}\right]+2\right)\left(\left[\frac{m}{2}\right]+1\right)-\left(\left[\frac{m}{2}\right]-n+1\right)\left(\left[\frac{m}{2}\right]-n+2\right)\right]=n\left[\frac{m}{2}\right]-\frac{n(n-3)}{2},
\end{array}
$$

by Descartes theorem of the Appendix, and we can choose the appropriate coefficients $A_{i, j}$ in order that the simple positive roots number of $S(r)$ is at most

$$
\mu_{2}=n\left[\frac{m}{2}\right]-\frac{n(n-3)}{2}-1=n\left[\frac{m}{2}\right]-\frac{n(n-3)+2}{2} .
$$

Hence, (b) of Theorem 3 is proved.

Example 1. We consider system (5), where $p=1, q=3$, and

$$
f(u, v)=2 u^{3}+u^{2} v-7.2365 u^{2}+5 v^{2}-0.5 u+0.7605 \text {. }
$$

In this case, $m=3, n=3$ and $\operatorname{Cs} \theta$ and $\operatorname{Sn} \theta$ are T-periodic functions with period $T=8.4131$. From equation (5), we obtain

$$
f^{0}(r)=-\frac{1}{T r}\left(A_{0.0}+A_{1.0} r^{2}+A_{0.1} r^{6}\right) .
$$

Upon using (16), we get

$$
\begin{aligned}
& \alpha_{0,0}=8.4131, \\
& \alpha_{2,0}=3.6276 \text { and } \alpha_{0,2}=2.1033 .
\end{aligned}
$$

So,

$$
f^{0}(r)=-\frac{1}{8.4131 r}\left(6.3982-26.251 r^{2}+10.517 r^{6}\right) .
$$

This polynomial has two positive real roots, $r_{1}=0.5$ and $r_{2}=1.2$. According to statement (a) of Theorem 3 , that system has exactly two limit cycles bifurcating from the periodic orbits of the center $\dot{u}=-v, \dot{u}=v^{5}$, using the averaging theory of first order. Figure 1 shows the limit cycles for Example 1.

\section{Appendix}

We remember Descartes' theorem regarding the real roots number of a real polynomial (for a proof, see, for example, [20]).
Descartes theorem: consider the following real polynomial:

$$
p(u)=a_{l_{1}} u_{l_{1}}+a_{l_{2}} u_{l_{2}}+\cdots+a_{l_{k}} u_{l_{k}},
$$

with $0 \leq l_{1}<l_{2}<\cdots<l_{k}$ and $a_{l_{1}} \neq 0$ real constants for $i \in\{1,2,3, \ldots, k\}$. Since $a_{l_{i}} a_{l_{i+1}}<0$, it can said that $a_{l_{i}}$ and $a_{l_{i+1}}$ admit a variation of sign. If the signs variations number of is $n$, then $p(u)$ admits at most $m$ positive real zeros. In aditios, it is always possible to pick out the coefficients of $p(u)$, where $p(u)$ admits exactly $k-1$ positive real zero.

\section{Data Availability}

No data were used to support this study.

\section{Conflicts of Interest}

This work does not have any conflicts of interest.

\section{Acknowledgments}

The fifth author extends their appreciation to the Deanship of Scientific Research at King Khalid University for funding this work through a research group program under grant (R.G.P-2/1/42).

\section{References}

[1] D. Hilbert, "Nachrichten von der Gesellschaft der Wissenschaften Phys. Ki 5," Bulletin of the American Mathematical Society, vol. 8, pp. 437-479, 1902.

[2] S. Smale, "Mathematical problems for the next century," in Mathematics: Frontiers and Perspectives, pp. 271-294, American Mathematical Society, Providence, RI, USA, 2000.

[3] H. Liang and J. Huang, "On the uniqueness and expression of limit cycles in planar polynomial differential system via monotone iterative technique," Applicable Analysis, 2020, In press.

[4] A. Menaceur and S. Boulaaras, "A number of limit cycle of sextic polynomial differential systems via the averaging 
theory," Boletim da Sociedade Paranaense de Matemática, vol. 39, no. 4, pp. 181-197, 2021.

[5] J. Chavarriga, E. Saez Szántó, I. Szanto, and M. Grau, "Coexistence of limit cycles and invariant algebraic curves for a Kukles system," Nonlinear Analysis, vol. 59, no. 5, pp. 673-693, 2004.

[6] I. S. Kukles, "Sur quelques cas de distinction entre un foyer et un centre, (French) C. R. (Doklady)," Bulletin of the American Mathematical Society, vol. 42, pp. 208-211, 1944.

[7] J. Llibre and A. C. Mereu, "Limit cycles for generalized Kukles polynomial differential systems," Nonlinear Analysis: Theory, Methods \& Applications, vol. 74, no. 4, pp. 1261-1271, 2011.

[8] A. Makhlouf and A. Menaceur, "On the Limit cycles of a class of generalized Kukles polynomial differential systems via averaging theory," Journal of Differential Equations, vol. 2015, Article ID 325102, 10 pages, 2015.

[9] A. Menaceur, S. Boulaaras, S. Alkhalaf, and S. Jain, "Limit cycles of a class of polynomial differential systems bifurcating from the periodic orbits of a linear center," Symmetry, vol. 12, no. 8,15 pages, 2020.

[10] A. Lins, W. Melo, and C. C. Pugh, "On Liénard's equation," Lecture Notes in Mathematics, vol. 597, pp. 335-357, Springer, Berlin, Germany, 1977.

[11] C. Li and j. Llibre, "Uniqueness of limit cycles for Liénard differential equations of degree four," Journal of Differential Equations, vol. 252, no. 4, pp. 3142-3162, 2012.

[12] J. Llibre, A. C. Mereu, and M. A. Teixeira, "Limit cycles of the generalized polynomial Liénard differential equations," Mathematical Proceedings of the Cambridge Philosophical Society, vol. 148, no. 2, pp. 363-383, 2010.

[13] J. Llibre and A. Makhlouf, "Limit cycles of a class of generalized liénard polynomial equations," Journal of Dynamical and Control Systems, vol. 21, no. 2, pp. 189-192, 2015.

[14] Q. Jianyuan and S. Shuliang, "Limit cycles for a class of polynomial differential systems," Electronic Journal of Qualitative Theory of Differential Equations, vol. 9, pp. 1-9, 2016.

[15] A. Buică and J. Llibre, "Averaging methods for finding periodic orbits vai Brouwer degree," Bulletin of Mathematical Sciences, vol. 128, pp. 7-22, 2004.

[16] J. A. Sanders and F. Verhulst, Averaging Methods In Nonlinear Dynamical Systems, Vol. 59, Springer-Verlag, New York, NY, USA, 1985.

[17] F. Verhulst, Nonlinear Differential Equations and Dynamical Systems, Universitex, Springer-Verlag, Berlin, Germany, 1996.

[18] A. M. Liapunov, "Stability of motion. With a contribution by V. A. Pliss and an introduction by," Mathematics in Science and Engineering, vol. 30, 1966.

[19] A. Gasull and J. Torregrosa, "A new algorithm for the computation of the Lyapunov constants for some degenerated critical points," Nonlinear Analysis: Theory, Methods \& Applications, vol. 47, no. 7, pp. 4479-4490, 2001.

[20] I. S. Berezin and N. P. Zhidkov, Computing Methods, Vol. II, Pergamon, Oxford, UK, 1964. 